\title{
PROPIEDADES PSICOMÉTRICAS DE LA ESCALA SOBRE LA ACTIVIDAD DEL AMOR CONCRETO EN UNA MUESTRA DE JÓVENES DE UNA UNIVERSIDAD PARTICULAR DE TRUJILLO
}

\author{
PSYCHOMETRIC PROPERTIES OF THE SCALE FOR THE CONCRETE LOVE \\ ACTIVITY IN A SAMPLE OF YOUNG PEOPLE IN A PRIVATE UNIVERSITY OF \\ TRUJILLO
}

Nikolal Rodas V. ${ }^{1}$; Janeth Molina A.

Universidad Nacional Mayor de San Marcos, Lima, Perú

(RECIBIDO 05/07/2012, ACEPTADO 20/11/2012)

\begin{abstract}
RESUMEN
Continuando con la línea de investigación del enfoque denominado "amor concreto" (Rodas, 2011), se plantearon los siguientes objetivos: Primero, evaluar las propiedades psicométricas de la Escala sobre la Actividad del Amor Concreto en una muestra de jóvenes de ambos sexos que estudian en una Universidad Particular de Trujillo. Segundo, describir las características del amor en dichos jóvenes, comparando cómo se distribuye respecto al género y cuál es su relación con la edad.Se encontró que la escala cuenta con características aceptables de confiabilidad y validez. Por otro lado, la actividad amorosa más alta fue la compatibilidad y la menos desarrollada, la proyectividad; no se encontraron diferencias significativas del amor según el género, mientras que la edad no correlacionó de manera significativa con el amor. Finalmente se discuten los resultados.
\end{abstract}

Palabras claves: Amor concreto, escala, intimidad, compatibilidad, proyectividad, jóvenes.

\begin{abstract}
Following the line of research approach called "concrete love" (Rodas, 2011), it was proposed the following objectives: First, evaluate the psychometric properties of the scale on Concrete Love activity in a sample of young men and women who study in a private university in Trujillo. Second, describe the characteristics of love in those youths, comparing how it is distributed on gender and your relationship with age. It was found that the scale has acceptable characteristics of reliability and validity. On the other hand, the highest activity was compatibility and projectivity was the less activity developed. Itdid not find significant differences by gender, while age did not correlate significantly with love. Finally we discuss the results.
\end{abstract}

Keywords: Concrete love, intimicy, compatibility, projectivity, young, scale.

1 Psicólogo por la Universidad Nacional Mayor de San Marcos. Estudiante de la Maestría en Psicología Clínica y de la Salud E-mail: nikolairodas@gmail.com 


\section{INTRODUCCIÓN}

En las relaciones de pareja se suele escuchar: "Me gustas", "te quiero", "te amo" ¿Estas palabras serán casuales? ¿Indicarán algo? Para la teoría del amor concreto (Rodas, 2011, 2012), corresponden a niveles de unidad en el desarrollo de la relación; es decir, toda pareja empieza gustándose, continúa queriéndose y fortalece su vínculo amándose, aunque también puede darse el caso de que una relación empiece amándose, luego se quiera y después se empiece a gustar. El hecho es que estos tres factores se encuentran en mayor o menor medida en todas las relaciones de pareja y estarían indicando ciertas etapas en el proceso del amor. En cada una de estas etapas-factores, las personas emplean un conjunto de estrategias, denominadas "actividades del amor concreto". Así, la primera actividad, que indica que la pareja se "gusta" es la intimidad; por medio de la cual, se produce, en palabras de Costa y Serrat (1982), un "intercambio de conductas mutuamente gratificantes"; los miembros del vínculo amoroso expresan sentimientos, deseos, necesidades, emociones y afectos mediante comportamiento verbal y no verbal. La actividad psicológica que indica que la pareja se "quiere" es la compatibilidad; mediante la cual la pareja evalúa su grado de similitud y sus diferencias, demostrando el conocimiento que cada uno tiene del otro y de sí mismo, tales como las costumbres, gustos, preferencias, aspiraciones; hacen uso de sus competencias de comunicación y resolución de conflictos. El tercer tipo de actividad conductual, que refleja que la pareja se "ama", es la proyectividad; es decir, la planificación de un futuro juntos, la cual evidencia el nivel de compromiso, madurez y responsabilidad.

Es importante recalcar que si bien estas tres conductas amorosas (intimidad, compatibilidad y proyectividad) siempre están presentes, una es la que predomina dependiendo de la etapa del desarrollo de la pareja. Por ejemplo, en el momento de empezar una relación, el deseo de pasar tiempo con el otro ya es un "proyecto" implícito, que se presenta en forma de expectativas, ilusiones e idealizaciones; éstas se fortalecen en la actividad íntima, pero serán des-ilusiones y des-idealizaciones en la medida en que se sobrelleve la etapa crítica de la compatibilidad, que tiende a presentarse como "crisis de pareja"; luego de ello, comienzan los verdaderos proyectos explícitos y realistas. Así pues, la patología del amor se explicaría por el déficit o deterioro de alguno de estos tres tipos de actividad, o bien por un "desnivel" en lo que se da y lo que se recibe. De allí la importancia de estudiarlos $\mathrm{y}$ analizar sus relaciones.

Un estudio preliminar (Aliaga, Rodas y Guevara, 2010), en una muestra de 30 personas de orientación heterosexual, que acudían a un consultorio psicológico de una universidad nacional, se encontró que los componentes de intimidad, compatibilidad y proyectividad de la Escala sobre la Actividad del Amor Concreto (Escala AAC), arrojaron un Alfa de Cronbachalto, de .90, .83 y .87 respectivamente, mientras que el instrumento total obtuvo un Alfa de Cronbach de .94; estos datos indicaron una adecuada confiabilidad. Acerca de la validez de contenido, esta se obtuvo mediante el coeficiente V-Aiken, que resultó de $80 \%$ de aceptación por 
lo menos en cada ítem, de un total de 10 jueces; además, la correlación ítem-test corregida fue mayor a .4 (Sig. de .05). Sobre la validez de criterio, se optó por un índice de discriminación de grupos extremos (cuartil 1 y 3), desechando 17 ítems de compatibilidad y proyectividad que no lograron diferencias significativas (a un nivel de significación de .05). El instrumento final quedó con 36 ítems. Por otro lado, respecto al género, no se encontraron diferencias significativas en ningún tipo de actividad amorosa, aunque se apreció que la media de las mujeres fue ligeramente superior al de los varones.

En otra investigación (Rodas, 2011), se planteó, aumentando la muestra a 102 participantes del mismo consultorio, agregar evidencias de validez y de confiabilidad; así como, estudiar la asociación del amor con algunas variables, como género, tipo de relación, tiempo de relación, número de hijos e ingreso económico. Fueron personas de orientación heterosexual, que referían problemas en algún área de sus vidas, los jóvenes representaron el $20 \%$, los adultos el $78 \%$ y el $2 \%$ fueron adultos mayores. En cuanto a la validez de contenido, se realizó una crítica a las teorías psicológicas y a los antecedentes de estudios sobre el amor para rescatar ítems importantes, incorporando cuatro enunciados a la versión del anterior estudio. Se solicitó a 10 jueces que validaran la escala, observándoseque los coeficientes $\mathrm{V}$ de Aiken oscilaron entre .9 y $1(\mathrm{p}<.05)$, al mismo tiempo, se aplicó la correlación ítem-test corregida, hallándose que sus coeficientes fueron considerables (mayores a .20). La validez de criterio se calculó mediante la comparación de grupos extremos (Cuartil 1 y 3), rescatándose todas las oraciones de la Escala, lo que indicó que discriminan personas con actividad amorosa baja y alta (a un nivel de significación de .05). Es pertinente señalar que el componente más deteriorado fue la compatibilidad, luego la intimidad y por último la proyectividad. La evidencia de validez de constructo se evaluó con la correlación entre conceptos; de esta manera, entre intimidad y compatibilidad, se halló una correlación positiva considerable $(\mathrm{r}=.75, \mathrm{p}<.01)$; entre intimidad y proyectividad la correlación fue media $(\mathrm{r}=.68, \mathrm{p}<.01)$; y entre compatibilidad y proyectividad, la relación fue considerable $(\mathrm{r}=.72, \mathrm{p}<.01)$. La confiabilidad (Alfa de Cronbach) también fue alta, quedando de la siguiente manera: En intimidad .94, en compatibilidad $.84 \mathrm{y}$ en proyectividad .89; la escala total obtuvo .96. Se halló que existen diferencias significativas en el amor que demuestran varones y mujeres, siendo la media de los primeros más alta que la de ellas en compatibilidad y proyectividad, en amor total, pero no en intimidad.

Estos dos estudios fueron realizados con muestras de una población clínica; es decir, personas con disfuncionalidad en su relación o en su familia (infidelidad, violencia, problemas conductuales de los hijos, etc.) y que solicitaron ayuda profesional en un consultorio psicológico de una universidad nacional. Por tal motivo, continuando con la línea de investigación del amor concreto, es importante explorar las características psicométricas de la Escala sobre la Actividad del Amor Concreto en una población sin patología evidente, evaluando al mismo tiempo las asociaciones y comparaciones con algunas variables y grupos. La muestra elegida fue de un grupo de jóvenes universitarios. 
Muchas veces a una temprana edad, entre los 18 y 29 años, las personas no saben cómo afrontar una relación de pareja, que trae consigo nuevas emociones y nuevas experiencias. Para muchos, el gusto y la atracción física son interpretados como amor; otros no saben exactamente si lo que están experimentando es signo de amor; y están los/as que, a pesar de que su pareja los/as maltrata, le siguen preguntando reiteradamente si los/as ama. Así, las relaciones afectivas muchas veces se convierten en motivaciones personales, proyectos que se incorporan en los estilos de vida que obedecen a historias particulares. Por tal motivo, una educación del amor es necesaria, para ello es importante tener una línea base desde la cual partir.

Los objetivos de la presente investigación fueron: Primero, evaluar las propiedades psicométricas de la Escala sobre la Actividad del Amor Concreto en una muestra de jóvenes de ambos sexos que estudian en una Universidad Particular de Trujillo. Segundo, describir las características del amor en dichos jóvenes, comparando cómo se distribuye respecto al género y cuál es su relación con la edad.

\section{MÉTODO}

Diseño y tipo de investigación: El diseño que se empleó fue el transversal (Hernández, Fernández y Baptista, 2006), ya que los datos fueron recogidos en un solo momento de tiempo, de abril a mayo de 2011. Asimismo, según Alarcón (2008) el tipo de investigación fue correlacional, en sus modalidades descriptivas y de comparación de grupos contrastantes, examinando la variable del género; del mismo modo, se analiza la relación del amor con la edad.

Participantes: La técnica de muestreo fue intencional, registrándose las contestaciones de estudiantes de las carreras profesionales de Administración (23), Arquitectura (24), Medicina Humana (18), Contabilidad (08), Enfermería (04), Ingeniería (55), Marketing (04), Nutrición (07) y Psicología (09). En cuanto al género, fueron 72 mujeres y 60 varones. Las edades oscilaron entre 16 y 28 años de edad, con una media de 19, mediana 19 y moda 18 . Además, 103 participantes señalaron que estaban en una relación de "enamorados", 2 indicaron que su relación era de "convivencia" y 3 manifestaron que eran "esposos". Cabe recalcar que fueron 152 estudiantes; sin embargo, no todos cumplimentaron los datos requeridos.

Instrumento: Se administró la Escala sobre la Actividad del Amor Concreto (Escala AAC), descrita por Aliaga, Rodas y Guevara (2010) y desarrollada por Rodas (2011). Este instrumento evalúa las tres manifestaciones de amor según el modelo del Sistema del Amor Concreto. Así, la subescala de intimidad (18 ítems) mide los procedimientos objetivos por los cuales la persona expresa sus sentimientos a su pareja, informando que le gusta. La subescala de compatibilidad (10 reactivos), evalúa las formas objetivas por las cuales la persona demuestra que conoce a su pareja y que la quiere. Y el subcomponente de proyectividad (12 enunciados), examina cómo la persona se proyecta con su pareja, informando que la ama. Los enunciados están redactados en sentido positivo acerca del amor, por 
ejemplo: "acaricio a mi pareja" (ítem 1). El estilo de la escala es tipo Likert con cinco alternativas: Casi Nunca, Poco Frecuente, Regularmente, Muy Frecuente y Casi Siempre. Los valores que se les asignan es del 1 al 5 .

Procedimiento: Se tramitó el permiso respectivo para poder realizar la investigación. Mientras tanto, se capacitaba a una psicóloga para administrar el instrumento. Respondieron la escala solo los jóvenes universitarios que decidieron participar de la investigación, algunos optaron por no responder todos los datos solicitados, por ejemplo la carrera universitaria, su edad, su nombre. Se les mencionó que si ellos lo requerían podían comunicarse con los autores del estudio para darles sus resultados y orientarles en las interrogantes que se pudieron haber suscitado por el hecho de responder el test. Las condiciones de administración del instrumento psicométrico, en cuanto a instrucciones y ambiente (aula de clase y luz natural) fueron similares para los participantes.

\section{RESULTADOS}

Para tomar decisiones en lo que respecta a la prueba estadística a utilizar, es necesario saber la normalidad. En la Tabla 1, se puede constatar que las puntuaciones en intimidad, compatibilidad y la escala total se distribuyen con normalidad, pero proyectividad y edad no. Por tal motivo, se decidió emplear el Coeficiente de Correlación Rho de Spearman para los análisis que involucren estas variables.

Tabla 1:Prueba de Normalidad para los tipos de actividad del amor concreto y edad

\begin{tabular}{cccccc}
\hline & Intimidad & Compatibilidad & Proyectividad & Escala Total & Edad \\
\hline $\begin{array}{c}\text { Kolmogorov- } \\
\text { Smirnov Z }\end{array}$ & .76 & 1.32 & 1.57 & .97 & 2.06 \\
Sig. & .62 & .06 & .01 & .30 & .00 \\
\hline
\end{tabular}

\section{Propiedades psicométricas:}

Confiabilidad: En la Escala total el Coeficiente Alpha de Cronbach resultó .95. En la subescala intimidad este coeficiente fue .91. En el subcomponente compatibilidad, el mismo coeficiente fue .87 . Y en el factor proyectividad se obtuvo.89 (Tabla 2). Estos datos sugieren que la Escala AAC cuenta con niveles altos de confiabilidad, lo cual significa, según Santisteban (2009) que el test es preciso como instrumento de medida.

Tabla 2: Coeficientes de confiabilidad Alpha de Cronbach

\begin{tabular}{ccccc}
\hline & Intimidad & Compatibilidad & Proyectividad & Escala Total \\
\hline Alpha de Cronbach & .91 & .87 & .89 & .95 \\
\hline
\end{tabular}


Evidencias de validez: Se optó por las formas de validez de contenido y de constructo. La validez de contenido se examinó mediante la correlación ítem-test corregida y el Alpha de Cronbach si se elimina cada ítem (Tabla 3).Se observó que las correlaciones de cada ítem con el total sin incluir el ítem son mayores a .4, a un nivel de significación de .05, indicando que los ítems aportan significativamente a la Escala total. Los ítems que más se orientan en esta dirección son, por ejemplo, el 6 ("Le expreso afectuosamente lo que siento por él/ella"), el 23 ("Me esfuerzo para que exista una comunicación constante") y el 34 ("Le propongo hacer proyectos de pareja").

Tabla 3: Coeficientes de correlación ítem-test corregido (r) y Alpha de Cronbach si se elimina cada ítem (Alpha). $\mathrm{p}<.05$

\begin{tabular}{|c|c|c|c|c|c|c|c|}
\hline $\begin{array}{l}\text { Sub } \\
\text { Escala }\end{array}$ & Ítem & $r$ & Alpha & & Ítem & $r$ & Alpha \\
\hline \multirow{22}{*}{$\begin{array}{l}\text { 足 } \\
\stackrel{\text { 妾 }}{\text { 点 }}\end{array}$} & 1 & .49 & .91 & \multirow{10}{*}{ 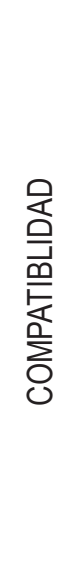 } & 19 & .57 & .85 \\
\hline & 2 & .59 & .91 & & 20 & .53 & .86 \\
\hline & 3 & .48 & .91 & & 21 & .50 & .86 \\
\hline & 4 & .56 & .91 & & 22 & .64 & .85 \\
\hline & 5 & .64 & .91 & & 23 & .73 & .84 \\
\hline & 6 & .70 & .91 & & 24 & .60 & .85 \\
\hline & 7 & .63 & .91 & & 25 & .56 & .85 \\
\hline & 8 & .58 & .91 & & 26 & .57 & .85 \\
\hline & 9 & .64 & .91 & & 27 & .64 & .85 \\
\hline & 10 & .63 & .91 & & 28 & .49 & .86 \\
\hline & 11 & .60 & .91 & \multirow{12}{*}{ 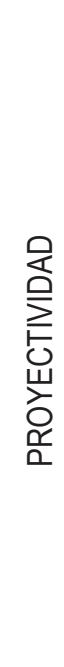 } & 29 & .46 & .88 \\
\hline & 12 & .65 & .91 & & 30 & .58 & .88 \\
\hline & 13 & .64 & .91 & & 31 & .67 & .87 \\
\hline & 14 & .48 & .91 & & 32 & .65 & .88 \\
\hline & 15 & .61 & .91 & & 33 & .65 & .88 \\
\hline & 16 & .60 & .91 & & 34 & .68 & .87 \\
\hline & 17 & .52 & .91 & & 35 & .55 & .89 \\
\hline & \multirow[t]{5}{*}{18} & \multirow[t]{5}{*}{.65} & \multirow[t]{5}{*}{.91} & & 36 & .62 & .88 \\
\hline & & & & & 37 & .66 & .87 \\
\hline & & & & & 38 & .61 & .88 \\
\hline & & & & & 39 & .58 & .88 \\
\hline & & & & & 40 & .53 & .89 \\
\hline
\end{tabular}


En cuanto a la validez de constructo, se optó por la modalidad de correlación entre conceptos, acerca de la cual Hernández, Fernández y Baptista (2006), sostuvieron que las mediciones entre los componentes deben estar asociadas entre sí, significando que se funden en un factor más general. En la Tabla 4 se aprecia que entre intimidad y compatibilidad existe una correlación directa considerable $(\mathrm{r}=.74$, $\mathrm{p}<.01$ ), entre intimidad y proyectividad se ha producido una asociación también directa y considerable $(\mathrm{r}=.71, \mathrm{p}<.01)$ y entre compatibilidad y proyectividad se encontró una correlación directa media $(\mathrm{r}=.67, \mathrm{p}<.01)$, siendo todas estas relaciones estadísticamente significativas.

Tabla 4: Coeficientes de correlación Rho de Spearman entre los tres tipos de actividad del amor concreto, Medias y Desviaciones Estándar

\begin{tabular}{ccll}
\hline Tipos de Actividad & Intimidad & Compatibilidad & Proyectividad \\
\hline Intimidad & 1 & & \\
Compatibilidad & $.74^{\star *}$ & 1 & \\
Proyectividad & $.71^{\star *}$ & $.67^{\star *}$ & 1 \\
\hline Media & 3.92 & 4.04 & 3.87 \\
Desviación Estándar & .61 & .60 & .69 \\
\hline
\end{tabular}

${ }^{* *} p<.01$ (dos colas) $/ \mathrm{n}=150$

\section{Características del amor concreto:}

\section{El amor en la muestra:}

En el gráfico 1, se observa que el tipo de actividad del amor concreto más desarrollado es la compatibilidad (media de 4.04) y el menos desarrollado es la proyectividad (media 3.87).

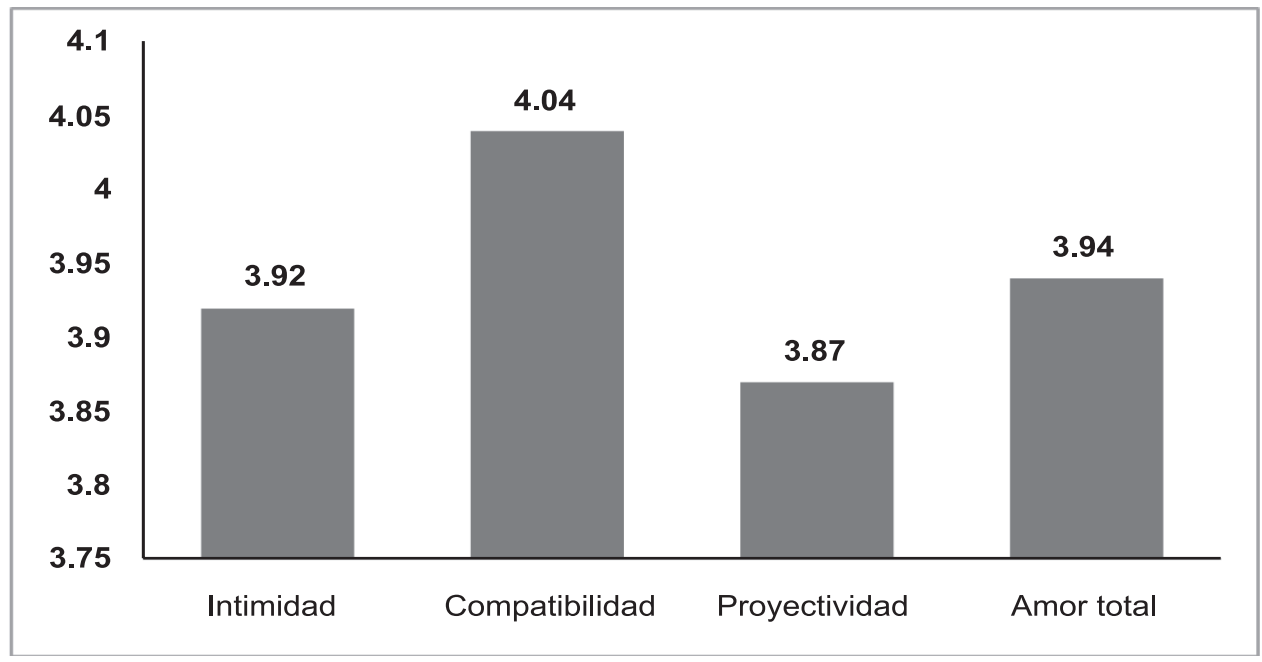

Gráfico 1: Niveles de intimidad, compatibilidad y proyectividad en jóvenes de una Universidad Particular de Trujillo 


\section{El amor según el género:}

En la Tabla 5 se puede apreciar que no existen diferencias significativas entre mujeres y varones en lo que respecta a los diferentes tipos de actividad del amor concreto (intimidad, compatibilidad y proyectividad) y el amor en su totalidad, a un nivel de significación de .05.

Tabla 5:Actividad del Amor Concreto según género

\begin{tabular}{|c|c|c|c|c|c|}
\hline $\begin{array}{l}\text { Tipos de Actividad del } \\
\text { Amor Concreto }\end{array}$ & Sexo & $\begin{array}{c}\text { Media del } \\
\text { Rango }\end{array}$ & Media & $\begin{array}{l}\text { U de Mann } \\
\text { Whitney }\end{array}$ & P-Valor \\
\hline \multirow{2}{*}{ Intimidad } & Mujer & 62.84 & 3.85 & \multirow{2}{*}{1896.500} & \multirow{2}{*}{$p=.23$} \\
\hline & Varón & 70.89 & 3.94 & & \\
\hline \multirow{2}{*}{ Compatibilidad } & Mujer & 64.88 & 3.99 & \multirow{2}{*}{2043.000} & \multirow{2}{*}{$p=.60$} \\
\hline & Varón & 68.45 & 4.06 & & \\
\hline \multirow{2}{*}{ Proyectividad } & Mujer & 66.04 & 3.84 & \multirow{2}{*}{2127.000} & \multirow{2}{*}{$p=.88$} \\
\hline & Varón & 67.05 & 3.87 & & \\
\hline \multirow{2}{*}{ AMOR total } & Mujer & 64.40 & 3.90 & \multirow{2}{*}{$2.008 \mathrm{E} 3$} & \multirow{2}{*}{$p=.49$} \\
\hline & Varón & 69.02 & 3.96 & & \\
\hline
\end{tabular}

$\mathrm{n}$ (Mujeres=72, Varones=30)

\section{Relación entre amor y edad:}

En la tabla 6 se puede observar que la correlación entre la edad y los tipos de actividad del amor concreto, incluyendo el amor total, es muy baja y estadísticamente no significativa $(\mathrm{p}>.01)$.

Tabla 6: Coeficientes de correlación Rho de Spearman entre el amor y la edad

\begin{tabular}{ccccc}
\hline Tipos de Actividad & Intimidad & Compatibilidad & Proyectividad & AMOR total \\
\hline Edad & .12 & .10 & .05 & .09 \\
\hline p>.01 (dos colas) $/ n=150$ La correlación no es significativa &
\end{tabular}

\section{DISCUSIÓN}

El análisis de los coeficientes de confiabilidad en el presente estudio y en los anteriores (Aliaga, Rodas y Guevara, 2010 y Rodas, 2011) sugieren que las puntuaciones son altas. Una explicación plausible es que se generó un adecuado rapport con los participantes, se les solicitaba que sus respuestas sean sinceras, ya que solamente de esa manera les podríamos ayudar, y calmábamos su ansiedad ante la "prueba" asegurándoles que no había respuestas buenas ni malas porque cada persona tiene un modo especial de expresar sus sentimientos. Por otro lado, el entorno donde se administró el instrumento fue iluminado, tranquilo y cómodo, donde las personas podían preguntar y opinar con confianza sobre el tema. 
Acerca de la validez de contenido, se pudo apreciar que los resultados de nuestra investigación fueron similares a los de Aliaga, Rodas y Guevara (2010) y Rodas (2011), evidenciándose una adecuada evidencia de validez de contenido. Una razón es que en los tres casos se revisaron teorías e investigaciones sobre el tema, se consultó con docentes de reconocida trayectoria en el área de pareja y de la psicometría, y se adecuaron los ítems partiendo de la experiencia clínica. Así, puede ir desarrollándose la idea de que la escala se constituye de reactivos que aportan significativamente en la contemplación del amor.

Sobre la evidencia de validez de constructo basada en la correlación de conceptos, en el estudio de Rodas (2011) y la presente investigación se halló una tendencia bastante cercana en la asociación entre intimidad y compatibilidad $(r=.74$ el 2011 y $\mathrm{r}=.75$ en la presente publicación); esto va ofreciendo mayor seguridad en la noción de que estos dos componentes forman parte de uno más grande o global que hemos denominado actividad del amor concreto. En cuanto a la intimidad y la proyectividad, en la primera investigación se halló una relación de .68, mientras que la actual encontró .74; es decir, aumentó en la muestra de jóvenes que no reportaron problemas graves en su relación, parece que en las relaciones disfuncionales la intimidad o está muy deteriorada o se la experimenta como una rutina que muy poco se integra en los proyectos. Finalmente, acerca de la compatibilidad y la proyectividad, el anterior estudio encontró $\mathrm{r}=.72$, mientras en la presente investigación disminuyó a .67; esto es, para que las parejas disfuncionales puedan mejorar su vínculo es necesario proyectarse en base a la compatibilidad; mientras que las relaciones de jóvenes sin problemas evidentes no se cuestionan demasiado ser compatibles, ya que la intimidad es para ellos una base para proyectarse con sus parejas.

Por otro lado, para la muestra con problemas en su relación (Rodas, 2011), la compatibilidad fue el tipo de actividad amorosa más deteriorado, seguido por la intimidad y la proyectividad. Por lo contrario, en el presente estudio la compatibilidad fue el componente más desarrollado, seguido por la intimidad y después la proyectividad. Esto pondría en evidencia el rol de la compatibilidad, o sea el respeto de las diferencias, la comunicación, la aceptación del otro y la resolución de conflictos en el desarrollo del amor. También, si queremos ir más allá, podríamos plantear la hipótesis de que la compatibilidad es el factor psicológico que determina el desarrollo saludable de una relación, así que tendría que ser fortalecida para que permita la expresión de sentimientos (intimidad) y la elaboración de proyectos (proyectividad). No obstante, tienen que realizarse más estudios con mayor diversidad en la muestra, puesto que en el presente, solo dos personas estuvieron en convivencia y tres en matrimonio, el resto estuvo en una relación de enamorados, lo cual hace que los datos hallados sean más característicos de este subgrupo.

El examen de la variable género, permite observar que en el presente estudio no se hallaron diferencias estadísticamente significativas entre varones y mujeres en lo que respecta a la expresión (intimidad), demostración (compatibilidad) y proyección (proyectividad) del amor. Sin embargo, Rodas (2011) halló que los varones presentan 
puntuaciones más altas y significativas en compatibilidad, proyectividad, amor total, pero no en intimidad. Además, Aliaga, Rodas y Guevara (2010) tampoco hallaron diferencias significativas. En esta última investigación, la muestra de personas con problemas en su relación fue 30, lo cual pudo influir en no hallar las diferencias reales, dado que sí se encontró que los varones tendían a presentar puntuaciones más altas, pero no estadísticamente significativas. Así que la comparación de nuestros resultados se aprecia mejor cuando se los analiza a la luz de la investigación de Rodas (2011). En la mencionada publicación, se había anotado que la muestra la conformaron parejas que asistían a un consultorio psicológico por diversos problemas, ya sea en sus relaciones amorosas o en sus contextos sociofamiliares, muchas de ellas fueron parejas disfuncionales. De aquella investigación se puede interpretar que los hombres después del inicio de la relación se descuidan, piensan que tienen asegurado el amor, disminuyen la frecuencia e intensidad de conductas amorosas hacia sus parejas e incluso pueden incurrir en relaciones extraconyugales o violencia familiar. Sin embargo, al ver que están perdiendo a aquella persona muy valiosa que es su pareja, comienzan nuevamente a incrementar sus conductas positivas; en cambio las mujeres, cansadas de dar y no recibir amor empiezan a esperar a que sea el hombre el que asuma la responsabilidad de desarrollar la relación, por ello disminuyen sus intercambios conductuales gratificantes. En la presente investigación, ya no en parejas disfuncionales, sino en jóvenes que no relatan problemas graves en sus relaciones, se halló que no existen diferencias significativas; o sea, hombres y mujeres aman por igual, estadísticamente hablando. De esta manera, los datos en abstracto indicarían que los hombres aman más que las mujeres; pero en concreto, los hombres aman más que las mujeres cuando perciben que la van a perder, las mujeres empiezan amar menos cuando no perciben reciprocidad por parte de sus parejas. Así, ingresaría un nuevo concepto, la reciprocidad, y se trataría de un elemento importante en toda la vida de la relación para que el amor se mantenga y se desarrolle. Este tema corresponde a otra investigación en curso. Por ahora se concluiría que, en situaciones "normales", tanto mujeres y varones expresan sentimientos de gusto del mismo modo, demuestran con la misma frecuencia que se quieren y se proyectan con la misma intensidad con la pareja.

Finalmente, acerca de la variable edad, nótese que las investigaciones anteriores con el concepto del amor concreto, no la consideraron en sus exploraciones. Según nuestros resultados, parece que "el amor no tiene edad". Cuando un "joven mayor", se enamora, lo puede hacer como un "joven menor". De allí que podría plantearse como hipótesis que no es la edad la que estaría determinando el sufrimiento o felicidad en las relaciones de pareja, sino tendrían que considerarse otras variables, por ejemplo, la experiencia previa en el ámbito del amor, la información sobre el tema, los modelos que se recibieron durante la infancia, rasgos psicológicos, etc.

\section{CONCLUSIONES}

1. En cuanto al primer objetivo, que indaga sobre las características psicométricas del test, se halló lo siguiente: 
- La confiabilidad fue adecuada. Así, en la escala total el coeficiente alpha de Cronbach resultó .95 , en la subescala intimidad este coeficiente fue .91, en el subcomponente compatibilidad el coeficiente fue $.87 \mathrm{y}$ en el factor proyectividad se obtuvo un coeficiente de .89 .

- La evidencia de validez de contenido indica que los ítems contribuyen significativamente a la escala total. Se empleó el método de la correlación ítem-test corregida y el Alpha de Cronbach si es que se elimina el ítem. Las correlaciones fueron mayores a .4, a un nivel de significación de .05 .

- Se halló adecuada evidencia de validez de constructo evaluada mediante las correlaciones entre los componentes de la Escala. Entre intimidad y compatibilidad existe una correlación directa considerable $(\mathrm{r}=.74, \mathrm{p}<.01)$, entre intimidad y proyectividad se ha producido una asociación también directa y considerable $(\mathrm{r}=.71$, $\mathrm{p}<.01)$ y entre compatibilidad y proyectividad se encontró una correlación directa media $(\mathrm{r}=.67, \mathrm{p}<.01)$, siendo todas estas relaciones estadísticamente significativas.

2. Acerca del segundo objetivo, que analiza comparaciones del amor en función del género y la edad, se encontró lo siguiente:

- El tipo de actividad del amor concreto más desarrollado es la compatibilidad (media 4.04), luego la intimidad (media 3.92) y después la proyectividad (media 3.87).

- No existen diferencias significativas entre mujeres y varones en lo que respecta a los diferentes tipos de actividad del amor concreto (intimidad, compatibilidad y proyectividad) y el amor en su totalidad.

- La correlación entre la edad y los tipos de actividad del amor concreto, incluyendo el amor total, es muy baja y estadísticamente no significativa.

\section{REFERENCIAS BIBLIOGRÁFICAS}

Alarcón, R. (2008). Métodos y diseños de investigación del comportamiento ( $2^{\mathrm{a}}$ ed.). Universidad Ricardo Palma. Editorial Universitaria: Perú.

Aliaga, J., Rodas, N. y Guevara, C. (2010). Análisis psicométrico de la Escala sobre la Actividad del Amor Concreto: Investigación preliminar. Nuevos Paradigmas, 4, 69-80.

Hernández, R., Fernández, C. y Baptista, P. (2006). Metodología de la investigación (4 ${ }^{\text {a }}$ Ed.). México: Mc Graw-Hill.

Rodas, N. (2011). Construcción y validación de la escala sobre la actividad del amor concreto. Tesis presentada para optar el título profesional de psicólogo en la Universidad Nacional Mayor de San Marcos. Perú.

Rodas, N. (2012). Introducción al paradigma del amor concreto. Revista de Investigación en Psicología, 14, 229-239

Santisteban. C. (2009). Principios de Psicometría. España: Síntesis S.A.

Costa, M. y Serrat, C. (1982). Terapia de parejas. España: Alianza Editorial. 